\title{
Effectiveness of combination of losartan potassium and doxycycline versus single-drug treatments in the secondary prevention of thoracic aortic aneurysm in Marfan syndrome
}

H. H. Clarice Yang, MSc, Jong Moo Kim, BSc, Elliott Chum, MD, Cornelis van Breemen, PhD, DVM, and Ada W. Y. Chung, PhD

Objective: Losartan potassium (INN losartan), an antihypertensive drug, has been shown to prevent thoracic aortic aneurysm in Marfan syndrome through the inhibition of transforming growth factor $\beta$. Recently we reported that doxycycline, a nonspecific inhibitor of matrix metalloproteinases 2 and 9, normalized aortic vasomotor function and suppressed aneurysm growth. We hypothesized that a combination of losartan potassium and doxycycline would offer better secondary prevention treatment than would single-drug therapy to manage thoracic aortic aneurysm.

Methods: A well-characterized mouse model of Marfan syndrome (Fbn1 $\left.{ }^{\mathrm{C} 1039 \mathrm{G} / /}\right)$ was used. At 4 months of age, when aneurysm had established, mice $(n=15 /$ group $)$ were given doxycycline alone $(0.24 \mathrm{~g} / \mathrm{L})$, losartan potassium alone $(0.6 \mathrm{~g} / \mathrm{L})$, or combined $(0.12-\mathrm{g} / \mathrm{L}$ doxycycline and $0.3-\mathrm{g} / \mathrm{L}$ losartan potassium) in drinking water. Littermate Fbn $1^{+/ t}$ mice served as control. Thoracic aortas at 6 and 9 months were studied.

Results: At 9 months, aortic diameter in untreated group was increased by $40 \%$ relative to control. Losartan potassium or doxycycline reduced aortic diameter by $10 \%$ to $16 \%$ versus untreated aortas. Losartan potassium and doxycycline combined completely prevented thoracic aortic aneurysm and improved elastic fiber organization, also downregulating matrix metalloproteinases 2 and 9 and transforming growth factor $\beta$ and normalizing aortic contractile and relaxation functions to control values.

Conclusions: Neither losartan potassium nor doxycycline alone completely restored vascular integrity and cell function when given during delayed treatment, indicating the importance of timed pharmacologic intervention. Combined, however, they synergistically offered better aneurysm-suppressing effects than did single-drug medication in the secondary prevention of thoracic aortic aneurysm. (J Thorac Cardiovasc Surg 2010;140:305-12)

Supplemental material is available online.

Marfan syndrome is an autosomal dominant disorder of connective tissue resulting from mutations in the gene encoding fibrillin-1. Fibrillin-1 is a principal component of the extracellular microfibrils, which are crucial for organizing scaffold in the formation and maturation of elastic fibers. Abnormal elastic fibers alter load-bearing capacity of the

\footnotetext{
From the Department of Cardiovascular Science, Child and Family Research Institute, University of British Columbia, Vancouver, British Columbia, Canada.

Supported by an operating grant from Canadian Institutes of Health Research. A.W.Y.C. is the recipient of Michael Smith Foundation for Health Research/ St Paul Hospital's Foundation Trainee Award. H.H.C.Y. is the recipient of Michael Smith Foundation for Health Research Junior Trainee Award and NSERC: Alexander Graham Bell Canada Graduate Scholarship.

Disclosures: None.

Received for publication March 29, 2009; revisions received May 31, 2009; accepted for publication Oct 25, 2009; available ahead of print March 2, 2010.

Address for reprints: Ada W. Y. Chung, PhD, Cardiovascular Science, Room 2099, 950 28th W Ave, Vancouver, British Columbia, Canada V5Z 4H4 (E-mail: adawingyee@yahoo.ca).

0022-5223/\$36.00

Copyright (c) 2010 by The American Association for Thoracic Surgery doi:10.1016/j.jtcvs.2009.10.039
}

aorta and cause microdissection, degeneration, and fibrosis of the media in the aorta. Consequently, progressive thoracic aortic aneurysm (TAA), dissection, and rupture account for more than $90 \%$ of mortality in Marfan syndrome. ${ }^{1}$ Clinical management of TAA in Marfan syndrome aims to decrease the rate of aortic root dilatation and reduce the risk of dissection and rupture. $\beta$-Adrenoceptor blockade (atenolol, propranolol hydrochloride [INN propranolol]) has been advocated for preventive therapy. ${ }^{2}$ This treatment does not, however, prevent attainment of important clinical end points, including aortic regurgitation, surgery, aortic dissection, and death. $\beta$-Adrenoceptor blockade improves aortic elasticity in patients with modest aortic dilatation, but no benefit is observed in patients with marked dilatation. ${ }^{3,4}$ There is thus a need for the development of more specific and effective pharmacologic strategies for the management of TAA in Marfan syndrome.

Aortic aneurysm is characterized by impairment of vascular cell function, destruction of extracellular matrix integrity, and deterioration of vessel wall mechanical properties. We have previously demonstrated that the progression of TAA in Marfan syndrome is associated with upregulation of matrix metalloproteinases (MMPs) MMP-2 and MMP-9, which is concomitant with extensive degeneration of elastic fibers, 


\section{Abbreviations and Acronyms \\ MMP $=$ metalloproteinase \\ TAA $=$ thoracic aortic aneurysm \\ TGF- $\beta=$ transforming growth factor $\beta$ \\ TIMP $=$ tissue inhibitor of metalloproteinase}

alterations in aortic mechanical properties, endothelial dysfunction, and reduction of smooth muscle contractility. ${ }^{5-7}$ Importantly, we have suggested that doxycycline, through its inhibition of MMP-2 and MMP-9, offers better aneurysm-suppressing properties than atenolol in the mouse model of Marfan syndrome. ${ }^{5}$

Recent investigations have demonstrated that perturbation of transforming growth factor $\beta$ (TGF- $\beta$ )/Smad2 signaling contributes to the prominent clinical manifestations in Marfan syndrome. Antagonizing TGF- $\beta$ through TGF$\beta$-neutralizing antibodies or losartan potassium (INN losar$\tan$ ), an angiotensin II type 1 receptor antagonist, has been shown to prevent aortic elastic fiber degeneration, aortic root dilatation, mitral valve prolapse, alveolar sepatation, and skeletal muscle dysfunction in a mouse model of Marfan syndrome. ${ }^{8,9}$ The prolonged administration of losartan potassium as a pharmacologic strategy for the management of TAA in patients with Marfan syndrome is therefore considered to have great potential. A multicenter, randomized, clinical trial has been initiated for the comparison of the effectiveness of losartan potassium and atenolol in patients with Marfan syndrome. ${ }^{10}$

From the recent literature, it has become clear that both TGF- $\beta$ and MMP-2/MMP-9 signaling are crucial in mediating aortic pathogenesis in Marfan syndrome, and the potential cross talk between these signaling molecules has been widely demonstrated. ${ }^{11-16}$ Overexpression of TGF- $\beta$ and the concomitant upregulation of various MMPs and tissue inhibitors of metalloproteinase (TIMPs) have been suggested to be associated with increased apoptosis, impaired progenitor cell recruitment, and abnormal directional migration, all of which are likely to contribute to aneurysmal development in Marfan syndrome. ${ }^{11,12}$ The combination of losartan potassium and doxycycline is of great interest because this combination, by downregulating MMPs and TGF- $\beta$, may potentially provide synergistic benefits in the management of TAA.

This study was designed to investigate the potential synergistic effects of combining doxycycline and losartan potassium in the secondary prevention of TAA in Marfan syndrome. We have demonstrated that neither losartan potassium nor doxycycline alone could completely restore vascular integrity and cell function when given during the delayed treatment, indicating the importance of timed pharmacologic intervention; however, combined treatment with losartan potassium and doxycycline effectively modified the progression of TAA by downregulating MMP/TGF- $\beta$ activation, normalizing elastic fiber structure and aortic cellular function, and, most importantly, reducing aneurysmal size.

\section{MATERIALS AND METHODS}

\section{Experimental Animals and Tissue Preparation}

Heterozygous $\left(\mathrm{Fbn} 1^{\mathrm{C} 1039 \mathrm{G} / 4}\right.$, the most common class of mutation in Marfan syndrome) mice were bred with wild-type mice (C57BL/6) to generate control $\left(\mathrm{Fbn}^{\text {th }}\right.$ ) and Marfan mice, which were housed in the institutional animal facility. All animal procedures were approved by the institutional animal ethics board, ${ }^{5-8}$ and all animals received humane care in compliance with the Guide for the Care and Use of Laboratory Animals (www.nap.edu/ catalog/5140.html).

Two major questions were addressed: (1) How effective are losartan potassium and doxycycline in remedying TAA and vascular function when given alone after pronounced aneurysm has developed (4 months of $\left.\operatorname{age}^{5-8}\right)$ ? (2) What, if any, are the synergistic aneurysm-suppressing effects of combined therapy?

To highlight the synergistic relation between losartan potassium and doxycycline, we used half the dosage used in the primary treatment ${ }^{5,8}$ in the combined therapy. From preliminary experiments, we found that half the dosage of either drug alone did not have a significant aneurysm-suppressing effect and did not improve vasomotor function (data not shown). Marfan mice starting at 4 months of age were left untreated or given losartan potassium $(0.6 \mathrm{~g} / \mathrm{L}, \mathrm{n}=30),{ }^{8}$ doxycycline $(0.24 \mathrm{~g} / \mathrm{L}, \mathrm{n}=30),{ }^{5}$ or a combination of losartan potassium and doxycycline $(0.3-\mathrm{g} / \mathrm{L}$ losartan potassium plus $0.12-\mathrm{g} / \mathrm{L}$ doxycycline, $\mathrm{n}=30$ ) in drinking water, then humanely killed at 6 or 9 months. Because doxycycline is light sensitive and only stable in aqueous solution for 48 hours, the doxycycline-laced water was shielded from all light and changed every other day, while the losartan potassiumlaced water was changed once a week.

Segments of ascending thoracic aorta (length, $1.2 \mathrm{~mm}$ ), aortic arch (3 $\mathrm{mm})$, and descending thoracic aorta $(3 \mathrm{~mm})$ were fixed for histologic analysis. A segment of aortic arch $(1.8 \mathrm{~mm})$ was used for the functional study (ascending aorta was not used for functional study because of its short length). The remaining aortic arch was flash-frozen for sample homogenization and protein extraction.

Procedures for gelatinolytic zymography, Movat staining, isometric force measurement Western immunoblotting, and aortic mechanical property determination are provided in Appendix E1.

\section{Statistics}

Data are reported as mean \pm SEM. Statistical analysis and stress-strain exponential curves were prepared with GraphPad Prism software (GraphPad Software, Inc, San Diego, Calif). Comparisons were performed with 1-way analysis of variance (with Bonferroni post hoc analysis for multiple comparisons).

\section{RESULTS \\ Thoracic Aortic Aneurysm}

At 6 and 9 months of age, the ascending aorta was enlarged by $40 \%$ in the untreated Marfan mice compared with the age-matched controls. With losartan potassium treatment, aortic diameter was increased by $25 \%$ at 6 months but not significantly increased at 9 months relative to control. Aortic dilatation was significantly attenuated by doxycycline at 6 months of age; at 9 months, the aortic diameter in the doxycycline group was comparable to that in the 


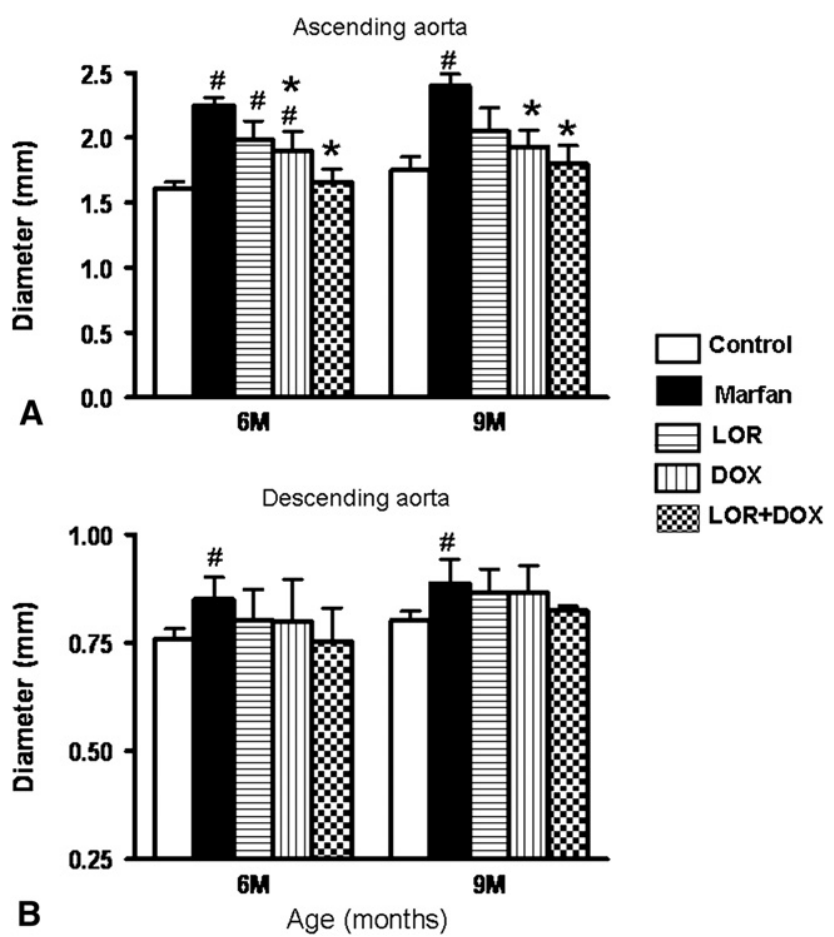

FIGURE 1. Average diameter thoracic aorta. A, Ascending; B, descending. Hatch mark indicates $P<.05$ versus age-matched control. Asterisk indicates $P<.05$ versus age-matched untreated Marfan group. LOR, Losartan potassium; $D O X$, doxycycline.

control group. Doxycycline and its combination with losartan potassium prevented aortic dilatation throughout the life span (Figure 1, $A$ ). There was descending thoracic aortic dilatation in the untreated Marfan group, which was prevented by both single and combined therapies (Figure 1, B).

\section{Elastic Fiber Structural Integrity}

Compared with control, Marfan aortic elastic fibers exhibited severe fragmentation, thinning, and disorganization. ${ }^{5,6}$ Losartan potassium and doxycycline improved the architecture, although breakage of elastic fibers was still observed within the media. Areas of local thinning, or uneven aortic wall thickness, were observed in the losartan potassium group. The combination of losartan potassium and doxycycline greatly improved the elastic fiber structure and restored the wavy organization, which was indistinguishable from fibers in control specimens (Figures 2, A, and 3).

To investigate the structural stability, we measured the breaking stress, which represents the breakage of the elastic fiber and smooth muscle association and at which the vessel could no longer maintain the stable resting tension. At 6 months, the breaking stress was 33\% lower in the untreated Marfan aortas than in aortas from the control mice; the ease of losing preservation of stable tension suggested structural weakening in the untreated aorta. Losartan potassium,

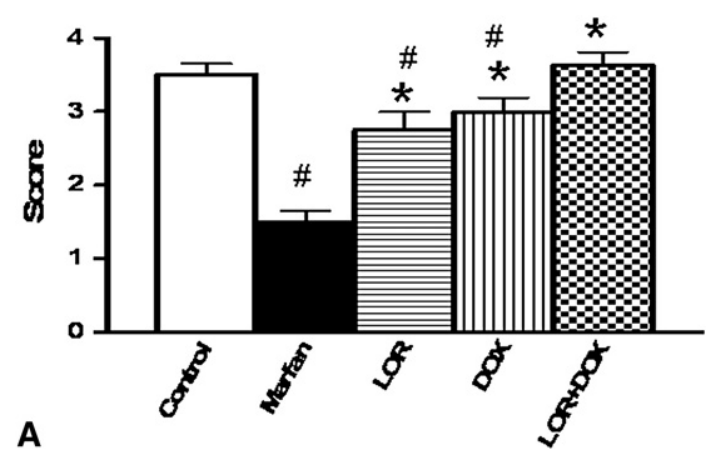

A

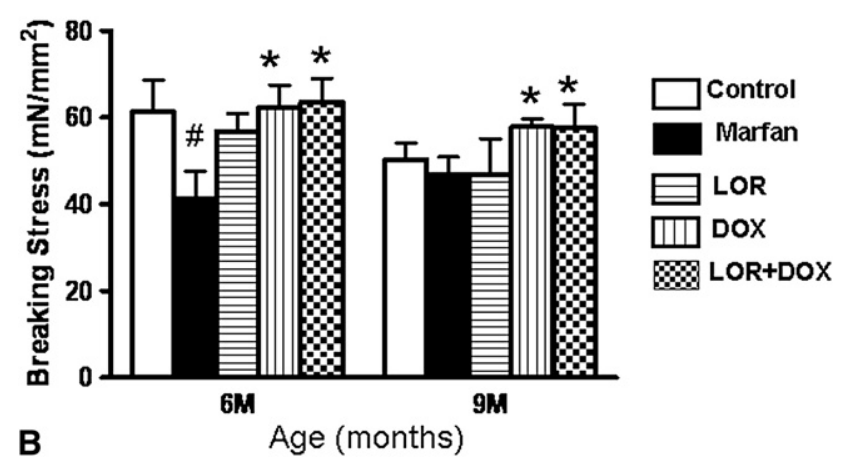

FIGURE 2. A, Averaged architecture score of aortic wall $(\mathrm{n}=7-10)$. Three aortic segments from each mouse ( 9 months) were examined by 3 observers blinded to genotype and treatment. B, Breaking stress of thoracic aorta. Hatch mark indicates $P<.05$ versus age-matched control. Asterisk indicates $P<.05$ versus age-matched untreated Marfan group. LOR, Losartan potassium; $D O X$, doxycycline.

doxycycline, and the combination of losartan potassium and doxycycline effectively improved breaking stress to the control value. At 9 months, although control, untreated, and losartan potassium-treated aortas showed similar breaking stresses, aortas treated with doxycycline and with combination of doxycycline with losartan potassium slightly but significantly increased breaking stress by $20 \%$ compared with the untreated value (Figure 2, B).

\section{Expressions of MMP-2, MMP-9, and TGF- $\beta$ / \\ Phosphorylated Smad2}

Although doxycycline prevented the upregulation of gelatinolytic activities of MMP-2 and MMP-9 at 6 months of age, pronounced enhancement of MMP activity at 9 months was not prevented by doxycycline. Whereas losartan potassium alone reduced the increased MMP-2 and MMP-9 activities only at 9 months of age, in combination with doxycycline it suppressed the MMP activation at both 6 and 9 months (Figure 4, A).

In the Western immunoblotting, the upregulation of MMP-9/TIMP-1 ratio in the Marfan group at 9 months of age was effectively prevented by losartan potassium both alone and in combination with doxycycline. At 6 months, the MMP-2/TIMP-2 ratio was significantly reduced by 


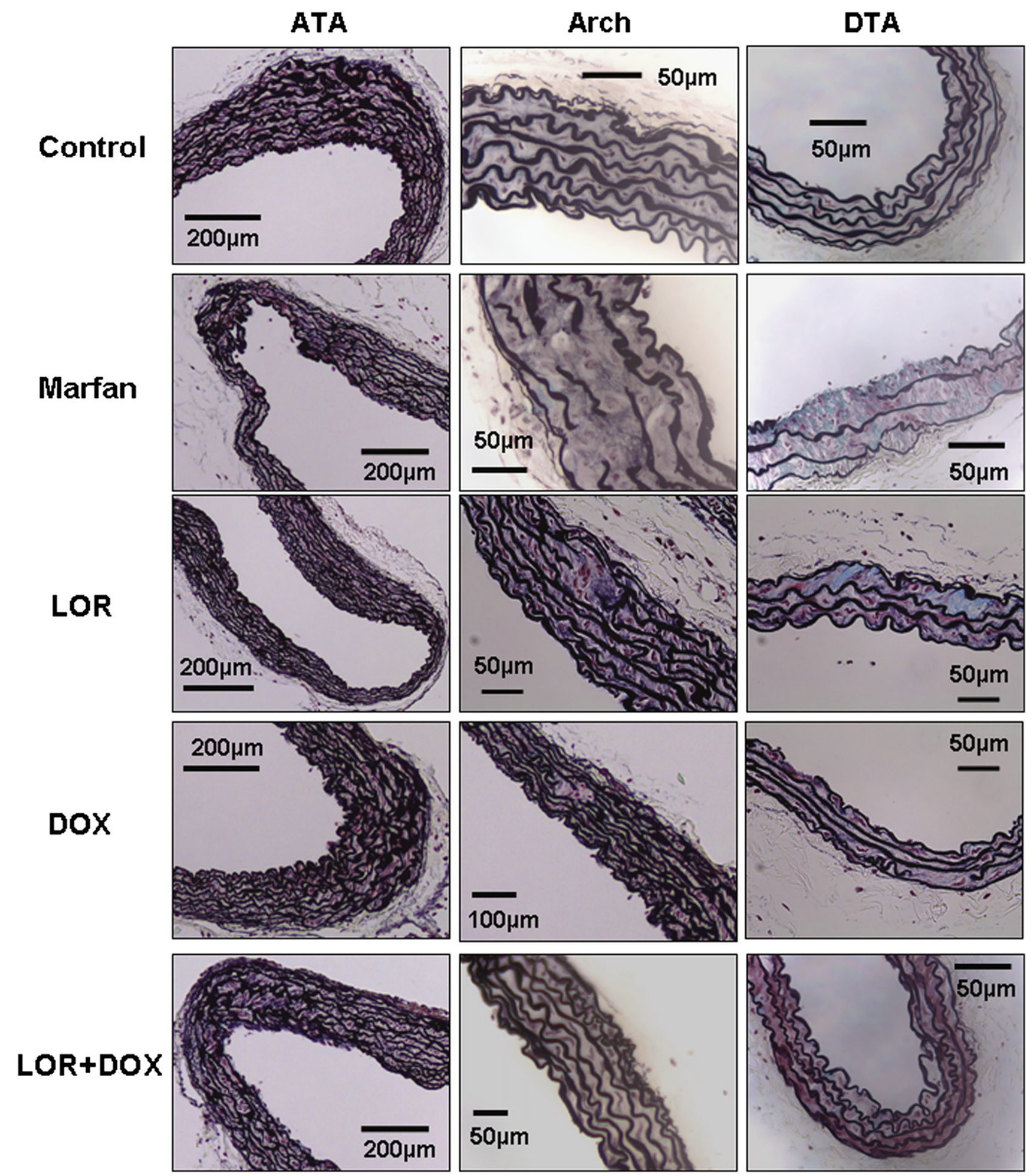

FIGURE 3. Integrity of elastic fibers examined on Movat-stained cross-sections of ascending thoracic aorta (ATA), arch, and descending thoracic aorta (DTA) from control, Marfan, Marfan with losartan potassium ( $L O R)$, Marfan with doxycycline (DOX), and Marfan with both drugs at 9 months. Elastic fibers are stained as black fibrils.

doxycycline, losartan potassium, and the combined treatment by $25 \%$ to $40 \%$ compared with the untreated group. At 9 months, losartan potassium suppressed the increase of MMP-2/TIMP-2 ratio in the Marfan aortas, and the combination with doxycycline further reduced this ratio to $70 \%$ of the control level (Figure 4, B). 

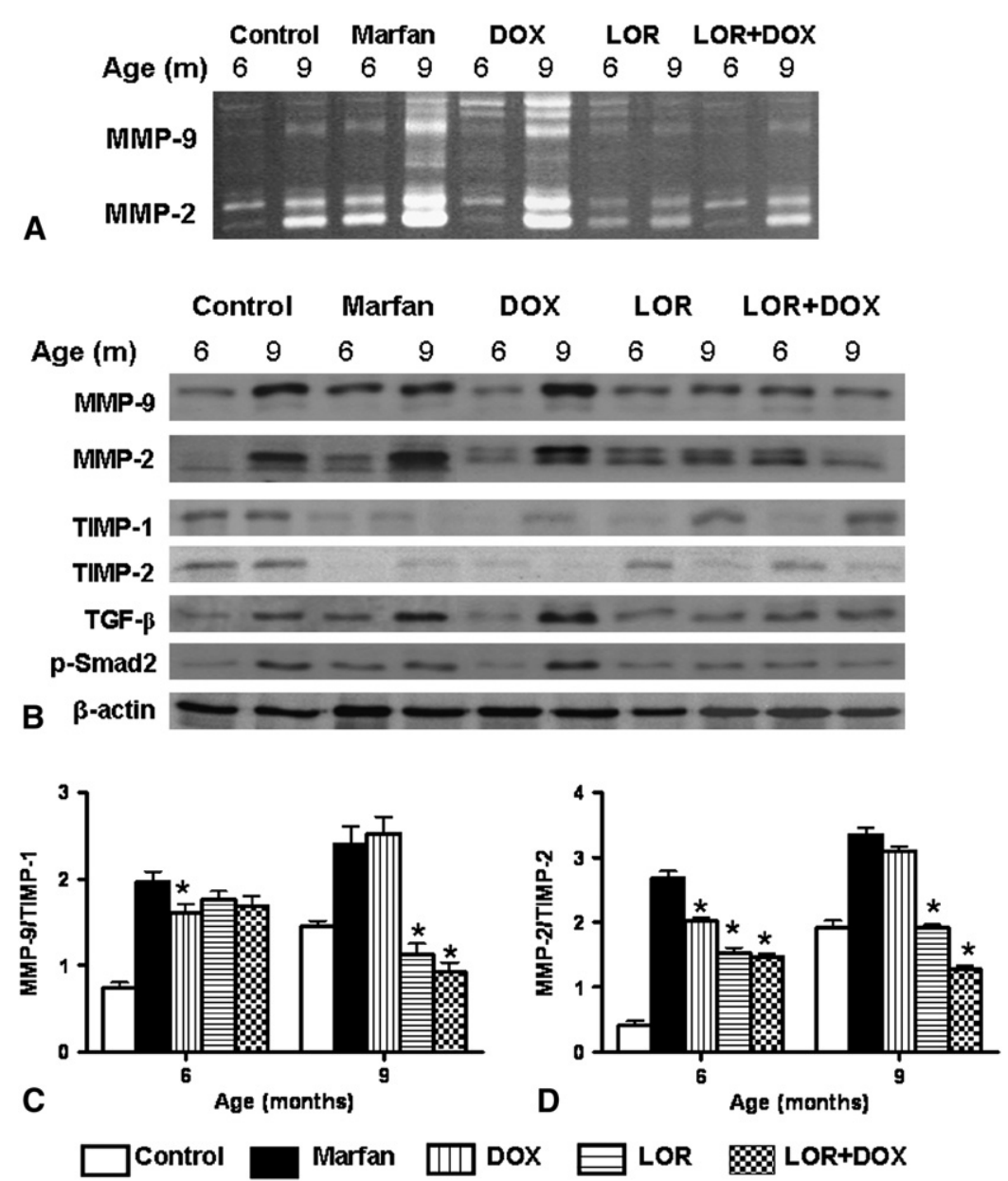

FIGURE 4. A, Representative gelatinolytic zymograms indicating activities of matrix metalloproteinases (MMPs) 2 and 9. B, Representative Western blots showing protein expressions of matrix metalloproteinases 9 and 2, tissue inhibitors of metalloproteinase (TIMPs) 1 and 2, transforming growth factor $\beta$ (TGF$\beta$ ), and phosphorylated Smad2 ( $p$-Smad2) in aortic samples. Densitometric analysis shows ratios of expressions of matrix metalloproteinase 9/tissue inhibitor of metalloproteinase 1 (C) and matrix metalloproteinase 2/tissue inhibitor of metalloproteinase 2 (D). Asterisk indicates $P<.05$ versus age-matched untreated Marfan group. LOR, Losartan potassium; DOX, doxycycline.

The expressions of TGF- $\beta$ and phosphorylated Smad2 were in concordance with MMP-2 and MMP-9 levels. Doxycycline inhibited the increased expression of TGF- $\beta$ / phosphorylated Smad 2 at 6 months of age; however, this expression was elevated at 9 months. Losartan potassium alone and in combination with doxycycline normalized the TGF$\beta /$ phosphorylated Smad2 expression to the control level at both 6 and 9 months of age (Figure 4,B).

\section{Vasocontractile Function and Endothelial-Dependent Relaxation}

At 6 and 9 months, the depressed potassium chloride-induced contraction in the Marfan aorta was normalized to the control values with losartan potassium, doxycycline, and combined treatment (Figure 5, $A$ and $B$ ). The depressed phenylephrine-stimulated contraction (in terms of both maximum effect and negative logarithm of $50 \%$ effective concentration values) in the untreated group at 9 months was improved by losartan potassium alone and in combination with doxycycline (Figure 5, $C$ and $D$ ).

The maximum effect of endothelium-dependent acetylcholine-induced relaxation was significantly improved by doxycycline at 6 months of age, although the response remained suppressed relative to the control value. Losartan potassium exhibited a trend toward improvement in relaxation that was comparable to the response of doxycycline, although it did not reach a significant difference from the untreated group (Figure 5,E). The negative logarithm of $50 \%$ effective concentration values of acetylcholine response was normalized to the control level by all treatments at 6 and 9 months (data not shown). At 9 months, control and untreated groups exhibited similar relaxation responses; however, doxycycline both alone and in combination with losartan potassium greatly enhanced the relaxation response, to $72 \%$ and $80 \%$, respectively (Figure 5, F). 

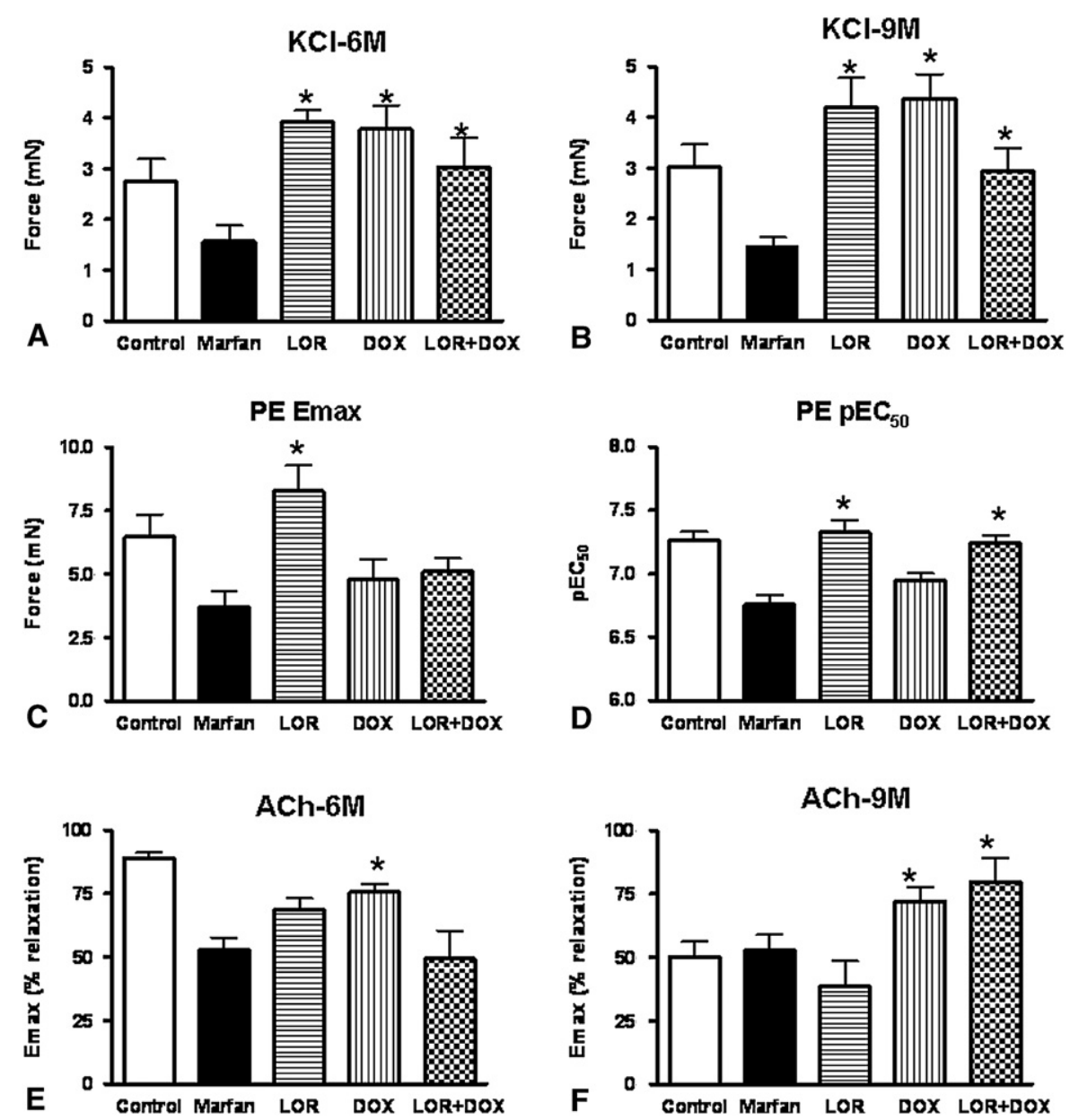

FIGURE 5. Contraction induced by $60-\mathrm{mmol} / \mathrm{L}$ potassium chloride ( $\mathrm{CCl}$, A and B). A, At 6 months; $\mathrm{B}$, at 9 months. Contraction stimulated by phenylephrine $(P E)$ at 9 months of age (C and D). C, Maximal response (Emax); D, Negative logarithm of $50 \%$ effective concentration $\left(p E C_{50}\right)$. Maximal relaxation induced by acetylcholine ( $A C h, \mathrm{E}$ and F). E, At 6 months; F, at 9 months. Asterisk indicates $P<.05$ versus age-matched untreated Marfan group. LOR, Losartan potassium; $D O X$, doxycycline.

\section{DISCUSSION}

Despite major advances in the medical and surgical management of Marfan syndrome, morbidity persists. Existing medical therapies, such as $\beta$-adrenoceptor blockers and calcium-channel blockers, reduce hemodynamic stress on the aorta $^{2}$ but do not target the pathogenetic basis of Marfan syndrome. Recent publications have demonstrated that deficiency of fibrillin-1-containing microfibrils results in excessive TGF- $\beta$ activation in many tissues that are altered in Marfan syndrome. TGF- $\beta$ antagonism by losartan potassium has been shown to prevent aortic root dilatation, mitral valve prolapse, lung disease, and skeletal muscle dysfunction in a mouse model of Marfan syndrome. ${ }^{8,9}$ We have also demonstrated the benefits of doxycycline, through its inhibition on MMP-2 and MMP-9, in preventing TAA, preserving elastic fiber integrity, and normalizing aortic vasomotor function and mechanical properties. ${ }^{5,6}$ Both TGF- $\beta$ and MMP-2 and MMP-9 are therefore believed to be crucial molecular mediators in the pathogenesis of
Marfan syndrome. This study has extended our previous findings and is the first to demonstrate the beneficial effects of losartan potassium and doxycycline in the secondary prevention of TAA in Marfan syndrome. There are 3 novel findings in this study: (1) Neither losartan potassium nor doxycycline alone could completely restore vascular integrity and cell function when given during the delayed treatment, indicating the importance of timed pharmacologic intervention. (2) During the delayed treatment, losartan potassium effectively improved aortic contractility, whereas doxycycline improved endothelium-dependent relaxation. (3) The combination of losartan potassium and doxycycline offered more beneficial effects than either single-drug therapy in improving elastic fiber organization, normalizing vasomotor function, inhibiting MMP-2, MMP-9, and TGF- $\beta$ activation, and, most importantly, reducing the size of aneurysm throughout the life span.

The secondary prevention study indicated the importance of the timing of pharmacologic intervention. We examined 
mice that were treated with doxycycline or losartan potassium once the process of aneurysmal degeneration had already become established. Our results indicated that even delayed treatment with either medication reduced aortic dilatation and improved elastic fiber architecture. Additionally, losartan potassium appeared to offer better contractile response and MMP and TGF- $\beta$ inhibitions and caused a moderate improvement in breaking stress, whereas doxycycline yielded better relaxation response and breaking stress threshold. This finding further supports the rationale for combined therapy. We have previously shown that the disruption of the MMP/TIMP balance could be associated with the excessive elastic fiber degradation in TAA., ${ }^{5,6}$ The remarkable reduction in MMP/TIMP ratios produced by losartan potassium both alone and in combination with doxycycline thus suggest that proteolytic activity in the vasculature and vascular remodeling during aortic dilatation could be reduced by these treatments. Furthermore, the normalization of the elastic fiber architecture by the combined treatment could contribute to the favorably increased breaking stress threshold, which is implicated in the reduced incidences of aortic dilatation, dissection, and rupture. During aging, the reduction of breaking stress in the control group demonstrates the detrimental effects of aging on vascular mechanical properties.

In the development of aneurysm, the increased proteinasemediated TGF- $\beta$ release could reinforce the proposed vicious cycle. ${ }^{6}$ We demonstrated that the losartan potassium both alone and in combination with doxycycline was effective in downregulating MMP activation in the secondary prevention. We previously reported the aneurysm-suppressing effects of doxycycline through the inhibition of MMP-2 and MMP-9, and the lack of inhibition of MMP expression in this study may have been due to the delayed intervention. The cross talk between MMP and TGF- $\beta$ has been extensively documented. TGF- $\beta$ overexpression and its downstream signaling (TGF- $\beta$ receptor/Smad) are associated with MMP upregulation at the transcriptional and translational levels, and MMP2 and MMP-9 have been identified as TGF- $\beta$ activators. ${ }^{11-16}$ Within the aortic wall during aging, TGF- $\beta$ activation is dependent on the age-associated increase in MMP-2 activity. ${ }^{15}$ Overexpression of TGF- $\beta$ and the concomitant upregulation of MMPs and TIMPs are associated with increased apoptosis, impaired progenitor cell recruitment, and abnormal directional migration, all of which are likely to contribute to aneurysmal development in Marfan syndrome. ${ }^{11,12}$ Increased TGF- $\beta$ signaling could cause valve calcification and dysfunction through the apoptotic events and increase in MMP-9. ${ }^{16}$ The homozygote dura stained for expression of activated TGF- $\beta$ and MMP-2 suggested that dural ectasia in MFS may be due to TGF- $\beta$ overactivation and to MMP-2-mediated elastolysis and collagen breakdown. ${ }^{13}$

Although in the delayed treatment neither losartan potassium nor doxycycline alone fully prevented vascular remodeling and cellular dysfunction, their combination greatly enhanced the beneficial effects of single-drug therapy by improving the aortic contractility and endothelium-dependent relaxation. The significant improvement in aortic contractility with the combined and losartan potassium-alone treatments could be explained by the normalization of elastic fiber integrity. It has been shown that growing smooth muscle cells on elastin preserves the contractile phenotype, as demonstrated by the presence of contractile myofilaments, and that mice deficient in elastin would develop less contractile force. ${ }^{17}$ According to ultrastructural analysis, elastic laminae connect to adjacent endothelial and smooth muscle cells through an intermediate structure composed of microfibrils. ${ }^{18}$ It has been shown that the arginine-glycine-aspartic acid sequence of fibrillin-1 can support cellular adhesion through integrin $\alpha_{5} \beta_{3}$, and these interactions are believed to contribute to the vascular structural integrity and to coordinate contractile and elastic tensions. ${ }^{19}$ Second, during the combined and the losartan potassium-alone treatment, the pronounced reduction of MMP could improve the contractility, because increased levels of MMP-2 and MMP-9 are associated with impaired contraction. ${ }^{5,6,20}$ Nevertheless, TGF- $\beta$ has been demonstrated to impair vasoconstriction directly. In mouse cerebral artery, high TGF- $\beta$ concentration inhibited endothelin-1-induced contraction by inducing mitogen-activated protein kinase phosphatase $1 .^{21}$ TGF- $\beta$ could cause vascular dysfunction of early diabetes by inhibiting calcium transients and impairing angiotensin II-induced contraction. ${ }^{22}$

Although losartan potassium exerted pronounced beneficial effects on aortic contractile function, it only moderately improved the endothelium-dependent relaxation. Through the transcriptional activation of the endothelial nitric oxide synthase gene promoter, TGF- $\beta$ and the downstream nuclear translocation of Smad-2 increase endothelial nitric oxide synthase expression and nitric oxide production in endothelial cells. ${ }^{23}$ Low blood level of TGF- $\beta$ is associated with the pathogenesis of atherosclerosis, vascular hypertrophy, and the severity of vascular disease. ${ }^{24}$ It is widely believed that nitric oxide mediates vasorelaxation, but it also regulates the balance of vascular smooth muscle cell proliferation and apoptosis, governing important aspects of vessel caliber and remodeling. ${ }^{25}$ The endothelial dysfunction during the losartan potassium treatment at 9 months of age thus may exert negative effects on vascular health and may be associated with aberrant vascular remodeling. Endothelial function could, however, be effectively enhanced with doxycycline. We have previously reported that doxycycline normalizes the endothelium-dependent relaxation and the basal nitric oxide level, coincident with the upregulation of endothelial nitric oxide synthase phosphorylation.$^{5-7}$ It is worthy of mention that aging caused endothelial dysfunction in both wild-type and untreated groups, which might mask the vascular pathogenesis of the disease. Aortas treated with doxycycline either alone or in combination with losartan potassium, however, exhibited better endothelial function even than the control. 
We acknowledge limitations of this study. First, the aortic diameter was assessed from the histologic slides rather than in vivo imaging, and this assessment does not allow the true measurement of the embedded aortic root. Lack of the in vivo longitudinal examination on aortic diameter does not allow us to confirm that either losartan potassium or doxycycline treatment offers aneurysm-suppressing benefits through the life span of the animal. This histologic assessment may also underestimate the difference in diameter. The differences in aortic diameter presented here might be more pronounced if we had performed in vivo measurements. Our results thus raise interesting questions for further testing with more sophisticated and appropriate methodology. Second, all functional experiments were performed on the aortic arch, but not the ascending part because of its very short length. We used the aortic arch protein in the molecular biology experiments, and the limited ascending aorta was fixed for histologic examination. Although most of the aneurysms in human patients with Marfan syndrome are seen in the aortic root and ascending aorta, our preliminary data and previous publications have shown that similar disease pathogenesis and aberrant signaling pathways are exhibited in the ascending aorta and the arch. ${ }^{5-7}$ Third, there are more than 600 mutations identified in Marfan syndrome. ${ }^{1}$ Whereas missense mutations account for $60 \%$ of the mutations, $78 \%$ of the point mutations are located in the collagen-binding epidermal growth factor (CBEGF) modules. A further $12 \%$ of these mutations are recurrent and affect a CpG mutation hotspot for a cysteine residue. ${ }^{1}$ This type of mutation is the basis of the mouse model used in this study and represents the most common mutation in classic Marfan syndrome. ${ }^{8}$ We therefore find that although our model is useful to investigate the general pathogenesis of Marfan syndrome, it may not be representative of all cases of Marfan syndrome in human beings.

In conclusion, we are the first to show that delayed treatment greatly reduces the effectiveness of single therapy with either losartan potassium or doxycycline, implicating the importance of timed pharmacologic intervention. In contrast, combined therapy with losartan potassium and doxycycline not only offered superior aneurysm protection but also improved vascular cell function and elastic fiber structural properties. Our study offers better understanding of optimal treatment for Marfan syndrome, which is important to lessen the risks of aortic dilatation, dissection, and rupture, and to reduce the associated morbidity and mortality.

\section{References}

1. Judge DP, Dietz HC. Marfan's syndrome. Lancet. 2005;366:1965-76.

2. Shores J, Berger KR, Murphy EA, Pyeritz RE. Progression of aortic dilatation and the benefit of long-term $\beta$-adrenergic blockade in Marfan's syndrome. $N$ Engl J Med. 1994;330:1335-41.

3. Rios AS, Silber EN, Bavishi N, Varga P, Burton BK, Clark WA, Denes P. Effect of long-term $\beta$-blockade on aortic root compliance in patients with Marfan syndrome. Am Heart J. 1999;137:1057-61.
4. Yin FC, Brin KP, Ting CT, Pyeritz RE. Arterial hemodynamic indexes in Marfan's syndrome. Circulation. 1989;79:854-62.

5. Chung AW, Yang HHC, Radomski MW, van Breemen C. Long-term doxycycline is more effective than atenolol to prevent thoracic aortic aneurysm in Marfan syndrome through the inhibition of matrix metalloproteinase-2 and -9. Circ Res. 2008;102:e73-85.

6. Chung AW, Au Yeung K, Sandor GG, Judge DP, Dietz HC, van Breemen C. Loss of elastic fiber integrity and reduction of vascular smooth muscle contraction resulting from the upregulated activities of matrix metalloproteinase- 2 and -9 in the thoracic aortic aneurysm in Marfan syndrome. Circ Res. 2007;101:512-22.

7. Chung AW, Au Yeung K, Cortes SF, Sandor GG, Judge DP, Dietz HC, et al. Endothelial dysfunction and compromised eNOS/Akt signaling in the thoracic aorta during the progression of Marfan syndrome. Br J Pharmacol. 2007;150: 1075-83.

8. Habashi JP, Judge DP, Holm TM, Cohn RD, Loeys BL, Cooper TK, et al. Losartan, an AT1 antagonist, prevents aortic aneurysm in a mouse model of Marfan syndrome. Science. 2006;312:117-21.

9. Neptune ER, Frischmeyer PA, Arking DE, Myers L, Bunton TE, Gayraud B, et al. Dysregulation of TGF- $\beta$ activation contributes to pathogenesis in Marfan syndrome. Nat Genet. 2003;33:407-11.

10. Matt P, Habashi J, Carrel T, Cameron DE, Van Eyk JE, Dietz HC. Recent advances in understanding Marfan syndrome: should we now treat surgical patients with losartan? J Thorac Cardiovasc Surg. 2008;135:389-94.

11. Nataatmadja M, West M, West J, Summers K, Walker P, Nagata M, et al. Abnormal extracellular matrix protein transport associated with increased apoptosis of vascular smooth muscle cells in Marfan syndrome and bicuspid aortic valve thoracic aortic aneurysm. Circulation. 2003;108 (1 Suppl):II329-34.

12. Ikonomidis JS, Jones JA, Barbour JR, Stroud RE, Clark LL, Kaplan BS, et al. Expression of matrix metalloproteinases and endogenous inhibitors within ascending aortic aneurysms of patients with Marfan syndrome. Circulation. 2006;114(1 Suppl):I365-70.

13. Jones KB, Myers L, Judge DP, Kirby PA, Dietz HC, Sponseller PD. Toward an understanding of dural ectasia: a light microscopy study in a murine model of Marfan syndrome. Spine. 2005;30:291-3.

14. Han YP, Tuan TL, Hughes M, Wu H, Garner WL. Transforming growth factor- $\beta-$ and tumor necrosis factor- $\alpha$-mediated induction and proteolytic activation of MMP-9 in human skin. $J$ Biol Chem. 2001;276:22341-50.

15. Wang M, Zhao D, Spinetti G, Zhang J, Jiang LQ, Pintus G, et al. Matrix metalloproteinase 2 activation of transforming growth factor- $\beta 1$ (TGF- $\beta_{1}$ ) and TGF$\beta_{1}$-type II receptor signaling within the aged arterial wall. Arterioscler Thromb Vasc Biol. 2006;26:1503-9.

16. Clark-Greuel JN, Connolly JM, Sorichillo E, Narula NR, Rapoport HS, Mohler ER 3rd, et al. Transforming growth factor- $\beta_{1}$ mechanisms in aortic valve calcification: increased alkaline phosphatase and related events. Ann Thorac Surg. 2007;83:946-53.

17. Karnik SK, Brooke BS, Bayes-Genis A, Sorensen L, Wythe JD, Schwartz RS, et al. A critical role for elastin signaling in vascular morphogenesis and disease. Development. 2003;130:411-23.

18. Davis EC. Smooth muscle cell to elastic lamina connections in developing mouse aorta. Role in aortic medial organization. Lab Invest. 1993;68:89-99.

19. Sakamoto H, Broekelmann T, Cheresh DA, Ramirez F, Rosenbloom J, Mecham RP. Cell-type specific recognition of RGD- and non-RGD-containing cell binding domains in fibrillin-1. J Biol Chem. 1996;271:4916-22.

20. Chew DK, Conte MS, Khalil RA. Matrix metalloproteinase-specific inhibition of $\mathrm{Ca}^{2+}$ entry mechanisms of vascular contraction. J Vasc Surg. 2004;40:1001-10.

21. Tong XK, Hamel E. Transforming growth factor- $\beta 1$ impairs endothelin-1-mediated contraction of brain vessels by inducing mitogen-activated protein (MAP) kinase phosphatase-1 and inhibiting p38 MAP kinase. Mol Pharmacol. 2007; 72:1476-83.

22. Sharma K, Deelman L, Madesh M, Kurz B, Ciccone E, Siva S, et al. Involvement of transforming growth factor- $\beta$ in regulation of calcium transients in diabetic vascular smooth muscle cells. Am J Physiol Renal Physiol. 2003;285:F1258-70.

23. Saura M, Zaragoza C, Cao W, Bao C, Rodríguez-Puyol M, Rodríguez-Puyol D, et al. Smad 2 mediates transforming growth factor- $\beta$ induction of endothelial nitric oxide synthase expression. Circ Res. 2002;91:806-13.

24. Grainger DJ, Kemp PR, Metcalfe JC, Liu AC, Lawn RM, Williams NR, et al. The serum concentration of active transforming growth factor $\beta$ is severely depressed in advanced atherosclerosis. Nat Med. 1995;1:74-9.

25. Schleicher M, Sessa WC. Are the mechanisms for NO-dependent vascular remodeling different from vasorelaxation in vivo? Arterioscler Thromb Vasc Biol. 2008; 28:1207-8. 


\section{Appendix E1. Additional Detail for Materials and Methods}

\section{Movat Staining}

Aortic segments were formalin fixed and embedded in paraffin. Because of the limited samples from each mouse, perfusion fixation was not performed. From each aortic sample, 3 cross-sections $(3 \mu \mathrm{m})$ were cut from the proximal end, prepared, and stained with modified Movat pentachrome. To ensure consistency and reproducible histologic procedures, all samples were fixed, cut, mounted, and stained by the same technician in the department of pathology. Image acquisition and processing were performed with a Nikon MicroPhot microscope (Nikon Instruments Inc, Melville, NY). Each slide was examined by 3 observers from the department of pathology who were blinded to genotype and treatment. On each slide, 8 regions were examined $\left(0^{\circ}, 45^{\circ}, 90^{\circ}\right.$, $135^{\circ}, 180^{\circ}, 225^{\circ}, 270^{\circ}, 315^{\circ}$ ) and given a score on a scale from 1 to 4 as follows: 1, extensive fragmentation and degradation; 2 , local degradation and fragmentation; 3 , mild disorganization without fragmentation; 4, completely intact with wavy organization. ${ }^{1-4}$ Aortic wall architecture score was averaged from these 8 regions, from 3 slides and 3 observers each. Lumen diameter was calculated assuming circular geometry as the inner vessel circumference divided by $\pi$.

\section{Gelatinolytic Zymography}

The procedures for gelatinolytic zymography have been extensively described in our previous publications. ${ }^{1,2,4,5}$ Briefly, aortic segments were ground with liquid nitrogen in a stainless-steel mortar and pestle. Tissue powder was mixed in 9 volumes of ice-cold lysis buffer $(50-\mathrm{mmol} / \mathrm{L}$ tris[hydroxymethyl]aminomethane [Tris] hydrochloride, $\mathrm{pH} 7.4$, with 3.1-mmol/L sucrose, 1-mmol/L dithiothreitol, $10-\mu \mathrm{g} / \mathrm{mL}$ leupeptin, $10-\mu \mathrm{g} / \mathrm{mL}$ soybean trypsin inhibitor, $2-\mu \mathrm{g} / \mathrm{mL}$ aprotinin, and $0.1 \%$ Triton X-100 [The Dow Chemical Company, Midland, Mich]). After 20 minutes of incubation on ice, samples were homogenized with a glass homogenizer. The gelatinolytic activity was analyzed by separating protein $(10 \mu \mathrm{g})$ on $8 \%$ sodium dodecyl sulfatepolyacrylamide gel electrophoresis gels copolymerized with gelatin $(2 \mathrm{mg} / \mathrm{mL})$ as substrate. After electrophoresis, the gels were washed with $2.5 \%$ Triton X-100 and then incubated in incubation buffer $(50-\mathrm{mmol} / \mathrm{L}$ Tris hydrochloride buffer with $0.15-\mathrm{mol} / \mathrm{L}$ sodium chloride, $5-\mathrm{mmol} / \mathrm{L}$ calcium chloride, and $0.05 \%$ sodium azide, $\mathrm{pH} 7.4$ ) at $37^{\circ} \mathrm{C}$ until activities of the enzymes could be determined. After incubation, the gels were stained with $0.05 \%$ Coomassie brilliant blue G-250 in a mixture of methanol/acetic acid/water (2.5:1:6.5) and destained in $4 \%$ methanol with $8 \%$ acetic acid. The gelatinolytic activity was identified as transparent bands against the background of Coomassie blue-stained gelatin. Images were taken with a Canon digital camera (Canon U.S.A., Inc, Lake Success, NY), and densitometry was analyzed with Quality One Image software (Bio-Rad Laboratories, Inc, Hercules, Calif).

\section{Western Immunoblotting}

Because of the limited sample from each mouse, aortic arch samples were pooled in groups with the same strain and age. Procedures of Western blotting were previously described. ${ }^{1-7}$ After homogenization, protein concentration was determined by Bradford reagent with bovine serum albumin as standards (Bio-Rad). Protein extracts ( $20 \mu \mathrm{g})$ were diluted to the equivalent amounts of total protein per sample in sample buffer containing $40 \%$ glycerol, $8 \%$ sodium dodecyl sulfate, $0.5-\mathrm{mol} / \mathrm{L}$ Tris hydrochloride ( $\mathrm{pH} 6.8$ ), $0.02 \%$ bromophenol blue, and $4 \% \beta$-mercaptoethanol. Samples were then subjected to $8 \%$ (for MMP-2 and MMP-9) or 13\% (for TIMP-1 and TIMP2), then transferred to polyvinyldifluoride membranes (Bio-Rad). Membranes were blocked overnight with blocking buffer $(5 \%$ nonfat dry milk in Tris-buffered saline solution containing $0.1 \%$ polysorbate 20 ) and then probed with antiMMP-2 and anti-MMP-9 antibodies (Siemens Healthcare Diagnostics Inc, Oncogene Science, Cambridge, Mass) and mouse monoclonal anti-TIMP-1 and anti-TIMP-2 antibodies (EMD Chemicals Inc, Gibbstown, NJ) overnight at $4^{\circ} \mathrm{C}$. After washing 3 times in Tris-buffered saline solution containing $0.1 \%$ polysorbate 20 , membranes were incubated with antimouse IgG peroxidase-conjugated secondary antibodies (dilution 1:2500) for 1 hour. The immunoreactive proteins were then visualized by chemiluminescence (GE Healthcare Life Sciences, Little Chalfont, UK), and densitometric measurement was performed. To ensure equal protein loading, membranes were stripped by incubating in $62.5-\mathrm{mmol} / \mathrm{L}$ Tris hydrochloride $(\mathrm{pH} 6.8), 1 \%$ sodium dodecyl sulfate, and $100-\mathrm{mmol} / \mathrm{L} \beta$-mercaptoethanol at $55^{\circ} \mathrm{C}$ and reprobed with anti- $\beta$-actin mouse antibody (dilution 1:5000). ${ }^{1-7}$

\section{Mechanical Properties of Aorta}

Vessel elasticity was deduced from the stress-strain exponential curves. ${ }^{1,2,8}$ The procedure was repeated until the vessel was unable to maintain its resting tension. The stress at which instability occurred was reported as breaking stress. The stress-strain relationship was graphed as J-shaped curves. Equation of the exponential growth was $\mathrm{Y}=$ Start $\cdot \exp (\mathrm{K} \cdot \mathrm{X})$, where $Y$ is stress, $X$ is strain, and $K$ is a rate constant at which $\mathrm{Y}$ increases exponentially. Increased $K$ value indicates increased stiffness.

\section{Isometric Force Measurement}

Aortic arch (uncurved part, $1.8 \mathrm{~mm}$ in length) was mounted on two 40- $\mu \mathrm{m}$ diameter stainless steel wires isometrically in a small-vessel myograph (DMT A/S, N Aarhus, Denmark) for the measurement of the generated force. Vessel segments were stretched for 30 minutes, at which time the resting force was stabilized, then challenged with $60-\mathrm{mmol} / \mathrm{L}$ potassium chloride. To evaluate the endothelium-dependent relaxation, 
vessels were precontracted with $3-\mu \mathrm{mol} / \mathrm{L}$ phenylephrine before addition of acetylcholine $(0.01 \mathrm{nmol} / \mathrm{L}-100 \mu \mathrm{mol} / \mathrm{L})$. Concentration-response curves of phenylephrine-induced contraction and acetylcholine-induced relaxation were constructed. The negative logarithm of the concentration of acetylcholine yielding half maximum response was assessed by linear interpolation on the semilogarithm concentrationresponse curve, $\mathrm{pEC}_{50}=-\log \left(\mathrm{EC}_{50}\right)$, so that $E C_{50}$ is the $50 \%$ effective concentration and $p E C_{50}$ is its negative logarithm. ${ }^{1,2,6,7,8}$

\section{Materials}

All other reagents were of the highest molecular grade, purchased from Sigma (Sigma-Aldrich Canada Ltd, Oakville, Ontario, Canada).

\section{References}

1. Chung AW, Yang HH, Radomski MW, van Breemen C. Long-term doxycycline is more effective than atenolol to prevent thoracic aortic aneurysm in Marfan syndrome through the inhibition of matrix metalloproteinase-2 and -9. Circ Res. 2008;102:e73-85.
2. Chung AW, Au Yeung K, Sandor GG, Judge DP, Dietz HC, van Breemen C. Loss of elastic fiber integrity and reduction of vascular smooth muscle contraction resulting from the upregulated activities of matrix metalloproteinase-2 and -9 in the thoracic aortic aneurysm in Marfan syndrome. Circ Res. 2007; 101:512-22.

3. Chung AW, Rauniyar P, Luo H, Hsiang YN, van Breemen C, Okon EB. Pharmacologic relaxation of vein grafts is beneficial compared with pressure distention caused by upregulation of endothelial nitric oxide synthase and nitric oxide production. J Thorac Cardiovasc Surg. 2006;132:925-32.

4. Chung AW, Rauniyar P, Luo H, Hsiang YN, van Breemen C, Okon EB. Pressure distention compared with pharmacologic relaxation in vein grafting upregulates matrix metalloproteinase-2 and -9. J Vasc Surg. 2005;42:747-56.

5. Chung AW, Hsiang YN, Matzke LA, McManus BM, van Breemen C, Okon EB. Reduced expression of vascular endothelial growth factor paralleled with the increased angiostatin expression resulting from the upregulated activities of matrix metalloproteinase- 2 and -9 in human type 2 diabetic arterial vasculature. Circ Res. 2006;99:140-8.

6. Chung AW, Au Yeung K, Cortes SF, Sandor GG, Judge DP, Dietz HC, et al. Endothelial dysfunction and compromised eNOS/Akt signaling in the thoracic aorta during the progression of Marfan syndrome. Br J Pharmacol. 2007;150: 1075-83.

7. Chung AW, Yang HH, van Breemen C. Imbalanced synthesis of cyclooxygenasederived thromboxane $\mathrm{A}(2)$ and prostacyclin compromises vasomotor function of the thoracic aorta in Marfan syndrome. Br J Pharmacol. 2007;152:305-12.

8. Chung AW, Yang HH, Au Yeung K, van Breemen C. Mechanical and pharmacological approaches to investigate the pathogenesis of Marfan syndrome in the abdominal aorta. $J$ Vas Res. 2008;45:314-22. 